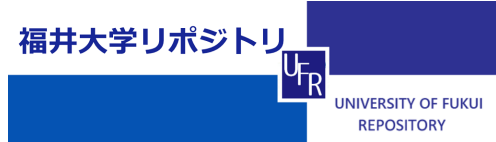

Negat i ve Absor pt i on Phenomenon I n Weakl y I oni zed Mer cury PI asma

\begin{tabular}{|l|l|}
\hline $\begin{array}{l}\text { j our nal or } \\
\text { publ i cat i on t i t l e }\end{array}$ & 福井大学工学部研究報告 \\
\hline vol une & 20 \\
\hline nunber & 2 \\
\hline page r ange & $169-179$ \\
\hline year & $1972-09$ \\
\hline URL & ht t p: //hdl . handl e. net /10098/4736 \\
\hline
\end{tabular}




\title{
Negative Absorption Phenomenon in Weakly lonized Mercury Plasma
}

\author{
T. IDEHARA, T. NODA, Y. IshIDA
}

(Received Apr. 13, 1972)

\begin{abstract}
Amplification of radio-frequency wave, which results from the negative absorption due to stimulated cyclotron radiation, is verified in weakly ionized mercury plasma. It does not occur continuously but does pulsively at certain phase of an If oscillation in plasma, and its maximum power gain at electron cyclotron frequency is estimated to be over $5 \mathrm{~dB}$ from experimental result.

The time-analyzed probe measurement shows that the excess of electrons is found in the energy region where the large Ramsauer effect is expected in the elastic collision cross section of electron, which is a necessary condition of negative absorption shown in theoretical consideration. If the parameters of plasma generation are adjusted to suitable values, the enormous emission pulse is observed synchronizing with an if oscillation, which is considered to be self-oscillation excited by any mechanism of feedback through plasma. Various features of this phenomenon is compared with those in the case of $\mathrm{Xe}, \mathrm{Kr}$ and Ar plasma.
\end{abstract}

\section{Introduction}

Possibility of negative absorption of electromagnetic wave in plasma was first predicted by Twiss, in order to explain the enormous radiation found in radio astronomy. ${ }^{1}$ Following his description, a necessary condition for the occurence of this phenomenon is that the kinetic energy distribution $f(\varepsilon)$ of the radiating electrons is markedly non-thermal with an appreciable excess of high energy electrons such that $\mathrm{df} / \mathrm{d} \varepsilon$ is positive over a finite range of the kinetic energy $\varepsilon$, where the stimulated transition probability should have a sharp maximum. This condition can be met for the cases in which the dominant radiation process is due (a) to Cerenkov effect, (b) to cyclotron radiation by non-relativistic electrons and (c) to synchrotron-type radiation by relativistic electrons.

After then, Bekefi et al. and Tanaka et al. discussed possibilities of negative absorption for various types of electron distribution function and stimulated transition probability..$^{2-5}$ As an example of them, it was described that the negative absorption due to stimulated cyclotron radiation and bremsstrahlung occurs in a partially ionized non-relativistic plasma, when population inversion of radiating electrons occurs, i. e.

\footnotetext{
* Department of Applied Physics
} 
$\mathrm{df} / \mathrm{d} \varepsilon$ is positive, in a range of electron energy where the Ramsauer effect is appreciable in the collision frequency $\nu$ for momentum transfer (which electron-neutral collision in weakly ionized plasma). ${ }^{2,4,5}$ If the Ramsauer effect and population inversion of electrons are described for mathematical convenience by expressions of $\nu(v) \propto v^{h}$ and $\mathrm{f}(\mathrm{v}) \propto \delta\left(\mathrm{v}-\mathrm{v}_{0}\right)$ (where $\mathrm{v}$ is electron speed), then the condition of negative absorption is $h>3$, which indicates that the large Ramsauer effect is expected for occurence of negative absorption.

Experimentally, an anomalous cyclotron emission pulse in microwave region was observed at a certain phase of low frequency oscillation in partially ionized $\mathrm{Xe}, \mathrm{Kr}$ and Ar plasma, and suggested to be a manifestation of the negative absorption due to stimulated cyclotron radiation predicted theoretically, since it does occur only in the plasmas having large Ramsauer effect. On the other hand, in order to give this phenomenon clear evidence, the amplifications of microwave and radio-frequency wave were verified in partially ionized $\mathrm{Xe}, \mathrm{Kr}$ and $\mathrm{Ar}$ plasma in the vicinities of electron cyclotron frequency and its harmonics $(f \approx n f)^{7,9,10}$ and the frequency region lower than collision frequency $(f<\nu) .{ }^{8}$ In the plasma produced by dc discharge, amplification as well as an anomalous emission was observed pulsively at a certain phase of If oscillation applied from external source, ${ }^{7-9}$ while in the neutral Xe gas penetrated by electron beam having low energy (several eV) which lies in the region of large Ramsauer effect, it does occur continuously at electron cyclotron frequency. ${ }^{10}$ These experiments verify completely the negative absorption due to the stimulated cyclotron radiation $(f \approx n f)$ and bremsstrahlung $(f<\nu)$ in a non-relativistic plasma.

In this paper, it is described that the negative absorption due to the stimulated cyclotron radiation is found also in mercury plasma where the Ramsauer effect is more conspicuous than $\mathrm{Xe}, \mathrm{Kr}$ and Ar plasma. In section 2, experimental apparatus and procedures are explained, in section 3 the experimental results and comparison of them with the theoretical consideration are presented and in section 4 the summaries of this paper are described.

\section{Experimental apparatus and procedures}

Through the experiment, we used a discharge tube (50 $\mathrm{mm}$ in inner diameter) filled with mercury vapor, which contains a tungsten mesh grid, a cylindrical probe and an anode disc $(30 \mathrm{~mm}$ in diameter) which are located $9 \mathrm{~mm}, 20 \mathrm{~mm}$ and $40 \mathrm{~mm}$ from the surface of an oxide-coated cathode $(25 \mathrm{~mm}$ in diameter $)$, respectively. In order to vary the pressure of mercury vapor $p$, the tube is put in the air bath whose temperature can be increased from $8^{\circ} \mathrm{C}$ to $120^{\circ} \mathrm{C}$, so that $\mathrm{p}$ is varied from $2.5 \times 10^{-4}$ to 0.7 Torr. A uniform magnetic field (its nonuniformity $\Delta \mathrm{B} / \mathrm{B}$ is smaller than one percent through the region of plasma) is applied parallel to the tube axis by the Helmholz coil, whose intensity is varied from 0 to 380 gauss. The plasma is produced by a dc discharge and an external low frequency voltage (several $\mathrm{KHz}$ ) is applied to anode in order to modulate dc discharge so that the plasma electrons may become 
far from the thermal equilibrium. The discharge current Id can be increased from $5 \mathrm{~mA}$ to $100 \mathrm{~mA}$ and the electron density varies from $2 \times 10^{8}$ to $9 \times 10^{9} \mathrm{~cm}^{-3}$ at pressure of $1 \mathrm{~m}$ Torr.

The experimental apparatus used here is shown in Fig. 1. A radio-frequency emission is picked up from the anode and detected by a field intensity meter (hereafter called FI meter) whose band width and minimum detectable power are about $0.08 \mathrm{MHz}$ and $2 \times 10^{-12}$ watt. A dc output of the FI meter is fed to a vertical axis of an $\mathrm{XY}$ recorder, while a magnetic field intensity to a horizontal axis, so that the emission intensity spectrum is displayed as a function of magnetic field intensity at

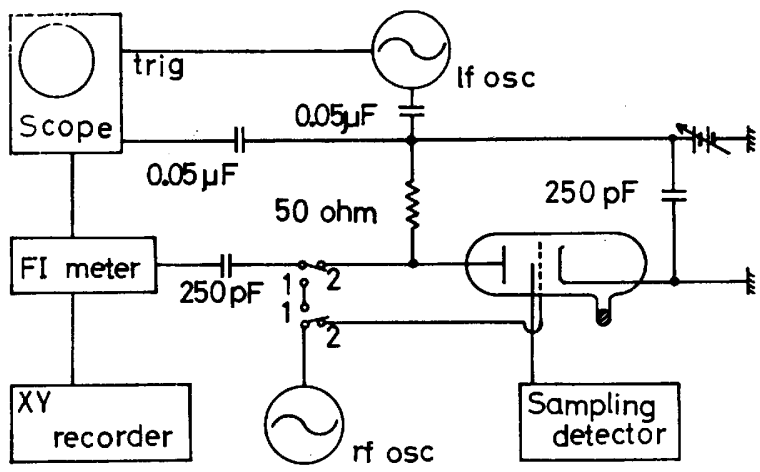

Fig. 1 Diagram of the experimental arrangement. a constant discharge current. On the other hand, the output voltage from intermediate frequency amplifier contained in FI meter (IF output voltage) is measured on a scope together with a low frequency oscillation picked up by the anode, in order to examine the phase relation between the rf emission and the if oscillation.

The negative absorption does not occur continuously but pulsively synchronizing with If oscillation as described in section 3. In order to examine plasma electron distribution at each phase of If oscillation and investigate the relation between the state of plasma and occurrence of negative absorption, the time-analyzed probe measurement is done, using the apparatus shown in Fig. 1. The current picked up by a probe situated at the center of plasma is fed to the sampling detector whose sampling pulse width is about one hundred nano second, and its dc output is displayed versus the probe voltage on an XY recorder at a constant discharge current Id and constant sampling time $T$, so that the characteristic curve of single probe at a definite phase of If oscillations is obtained. By doing the same measurement for various times $T$, we can obtain the characteristic curves for the corresponding phases of if oscillation.

In order to verify the negative absorption phenomenon obviously, we must examine the amplification of waves in plasma. When an rf signal is applied to the grid continuously, the output signal at the anode is detected by the FI meter and its IF output voltage $v_{2}$ is measured on a scope, while a voltage $v_{1}$ at the output of rf oscillator is directly picked up and its level is checked on a scope. On the other hand, the if oscillation at the anode is observed at the same time on a scope using another channel, so that the voltage ratio $\mathrm{v}_{2} / \mathrm{v}_{1}$ is estimated at each phase of If oscillation. In order to verify the amplification, we must estimate the power gain in plasma between grid and cathode $G=P_{2} / P_{g}$ and show that its value is larger than $0 \mathrm{~dB}$, where $P_{2}=v^{2}{ }_{2} / R_{2}$ is the output power at anode and $\mathrm{P}_{g}$ is the incident power to grid. However, it is difficult to determine the precise value of $\mathrm{G}$, because the output and input impedance 
$R_{2}$ and $R_{g}$ cannot be estimated precisely, so we will consider only the sufficient condition for amplification instead of estimation of $G$. Considering that the output impeadance of $\mathrm{rf}$ oscillator $\mathrm{R}_{1}$ is equal to $50 \mathrm{ohm}$, the gain $\mathrm{G}$ is described as follows.

$$
\begin{aligned}
\mathrm{G}=10 \log \left(\mathrm{P}_{2} / \mathrm{P}_{q}\right) & =10 \log \left(\mathrm{P}_{2} / \mathrm{P}_{1}\right)+10 \log \left(\mathrm{P}_{1} / \mathrm{P}_{g}\right) \\
& =20 \log \left(\mathrm{V}_{2} / \mathrm{V}_{1}\right)+10 \log \left(\mathrm{R}_{1} / \mathrm{R}_{2}\right)+10 \log \left(\mathrm{P}_{1} / \mathrm{P}_{0}\right)
\end{aligned}
$$

The third term of above equation is always positive because of mismatching at grid. Since we have set up to make $R_{2}=50 \mathrm{ohm}$, the second term is also positive, whatever value discharge impedance may take. Therefore, in our apparatus, the sufficient condition for amplification is that the first term is positive.

\section{Experimental results and discussions}

(a) Observation of pulsive emission near the electron cyclotron frequency

An enomous pulsive emission was observed near electron cyclotron frequency synch ronizing with if oscillation, whose feature is similar to that in the case of $\mathrm{Xe}, \mathrm{Kr}$ and Ar plasma, as shown below.

1) A series of emission spectra which were displayed on $X Y$ recorder as functions of normalized magnetic field $f_{c} / f$ with receiving frequency $f$ as a parameter shows an anomalously large peak near $f_{c} / f=1$, where $f_{c}$ is the electron cyclotron frequency. One of them is shown in Fig. 2 (a). The magnetic field $f_{c}$ where the emission is observed on the spectrum for each receiving frequency was plotted on $f_{c}$-f diagram, in Fig. 2 (b).

2) The feature of emission was observed on the lower trace of a scope together with if oscillation picked up at anode and fed to the upper trace. It is emitted as several ten micro second pulse, at a certain phase of $1 f$ oscillation, as shown in Fig. 3 .

3) The maximum power which we observed reaches $2 \times 10^{-6}$ watt. Considering that the normal cyclotron emission power cannot be over the blackbody emission power ${ }^{11}$ which is estimated to be about $10^{-12}$ watt in our plasma, the observed pulse emission power is anomalously large.

4) The width of frequency spectrum of the emission is several ten $\mathrm{MHz}$ and comparable with or smaller than the collision frequency of electron with the neutral atoms $\nu$, which

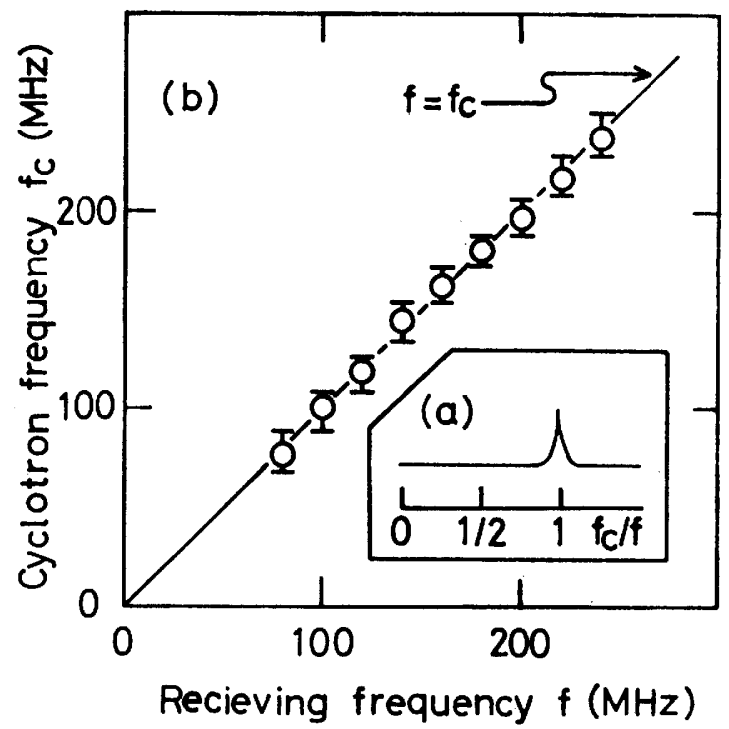

Fig. 2 (a) The emission spectrum as a function of normalized magnetic field $f_{c} / f$. $\mathrm{f}=180 \mathrm{MHz}$.

(b) The magnetic field where the anomalous emission apears is plotted versus receiving frequency. $\mathrm{p}=1.20 \mathrm{~m}$ Torr. $\mathrm{Id}=10.5 \mathrm{~mA}$ 
is calculated to be about $30 \mathrm{MHz}$ from our experimental condition. From the characters mentioned above, the emission is considered to be a manifestation of negative absorption due to the stimulated cyclotron radiation, which is verified in this section.

(b) Relation between a velocity distribution of electron and occurence of emission pulse

It may be interesting to examine the state of plasma when the emission pulse is observed. Noting that the pulse occurs at a certain phase of $1 f$ oscillation applied from external if oscillator, we do a probe measurement at a fixed phase of if oscillation using a sampling detector and examine plasma parameters at various phases over one period of If oscillation. Fig.4 (a) shows the rf emission signal and

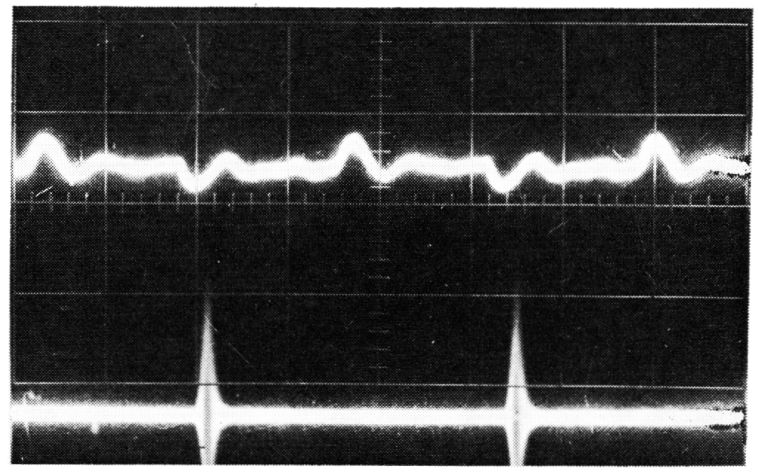

Fig. 3 Relation between an rf emission pulse and an lf oscillation voltage. Upper trace: an if oscillation voltage applied to an anode. 20 volt/div. Lower trace: an rf emission signal at the cyclotron frequency. The peak power is about $2 \times 10^{-7}$ watt. $\mathrm{f}=230 \mathrm{MHz} . \mathrm{f}_{c}=236 \mathrm{MHz}$. $\mathrm{p}=1.25 \mathrm{~m}$ Torr. $\mathrm{Id}=11.0 \mathrm{~mA}$. Sweep time: 0.1 $\mathrm{msec} / \mathrm{div}$.

If oscillation voltage both picked up at anode and displayed on a scope. The probe measurements were done at four different phases of if oscillation $T_{1}, T_{2}, T_{3}$ and $T_{4}$ shown in this figure, the results of which are drawn in Fig. 4 (b). The characteristic curve at $T_{1}$ shows that a plasma at this phase is nearly thermal, while the curves at $\mathrm{T}_{2}, \mathrm{~T}_{3}$ and $\mathrm{T}_{4}$ show the stepfeature and imply that the plasma at these phases is nonthermal and beam-like electrons exist in plasma. In Fig. 5, the beam energy which is determined by analyzing the characteristic curves is shown as a function of phase of If oscillation, together with electron density of background plasma electrons. The electron density varies with variation of lf oscillation voltage, delaying about 30 micro second. The beam energy varies from about $1 \mathrm{eV}$ to $2 \mathrm{eV}$ in the finite region of phase in a period. Comparing the beam energy with the emission signal shown on the lowest trace in this figure, the emission pulse is observed when the beam energy is found at about one electron volt. This value of beam energy does agree with that where the large Ramsauer effect is expected on the elastic electron collision cross section with the mercury atoms. ${ }^{12}$ This fact shows that the necessary condition for negative absorption discussed elsewhere ${ }^{2,4,5}$ is satisfied at the phase where the emission pulse is observed.

(c) Verification of the negative absorption of rf signal

In order to verify the negative absorption in plasma, it must be shown that rf signal applied from an external source is amplified in plasma when the enormous emission pulse mentioned above does not occur spontaneously. After the parameters of plasma generation (i. e. discharge current, if oscillation frequency and voltage and background pressure) were adjusted at suitable values so that the emission pulse was suppressed, 


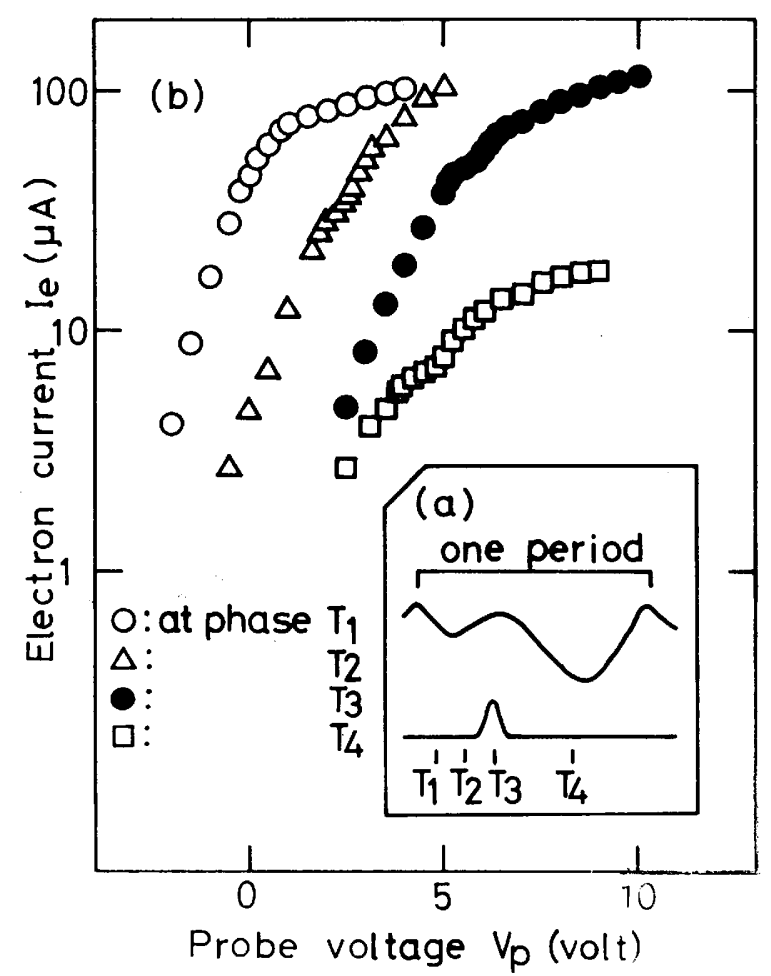

Fig. 4 The time-analyzed probe measurement at the various phase in the If oscillation.

(a) Upper trace: If oscillation voltage. The peak to peak voltage is 7 volt. Lower trace : $\mathrm{rf}$ emission signal. $\mathrm{f}=371 \mathrm{MHz} . \mathrm{f}_{c}=$ $371 \mathrm{MHz}$. One period is $290 \mu \mathrm{sec}$. Probe measurement were done at the phases denoted by $T_{1}, T_{2}, T_{3}$ and $T_{4}$.

(b) Characteristic curve of probe measurement at each phase. $\mathrm{p}=1.83$ mTorr. $\mathrm{Id}=4.0 \mathrm{~m} . \mathrm{A}$.

rf signal whose frequency $f$ is equal to the electron cyclotron frequency $f_{c}$ was applied to grid and the output signal at the anode was detected by FI meter and observed on a scope, using the apparatus shown in Fig. 1. In Fig. 6, the upper trace shows the if oscillation voltage at the anode, while the lower one shows both rf output siganls with and without input signal. Comparing the output signal level $v_{2}$ with the signal level at the output of $\mathrm{rf}$ oscillator $\mathrm{v}_{1}$, it is noted that the former is over the latter at the finite phase $T_{1}$ of lf oscillation where the fully suppressed emission pulse occurs spontaneously. In the case shown in this figure, the voltage ratio $\mathrm{v}_{2} / \mathrm{v}_{1}$ is about $4 \mathrm{~dB}$. From the consideration in the previous section, this result shows that the power of rf signal applied to the grid was amplified in plasma at the phase $T_{1}$.

The variation of the voltage ratio $v_{2} / v_{1}$ as a function of the power $P_{1}$ of rf oscillator was measured for various phases of if oscillation. The phases $T_{1}, T_{2}, T_{3}$ and $T_{4}$ where the measurement was done and the result of measurement at each phase is shown in Fig. 7 (a) and (b), respectively. At the phases $T_{2}, T_{3}$ and $T_{4}$, the ratios $v_{2} / v_{1}$ had negative values nearly independent on $P_{1}$, which were about $-13,-18$ and -20 


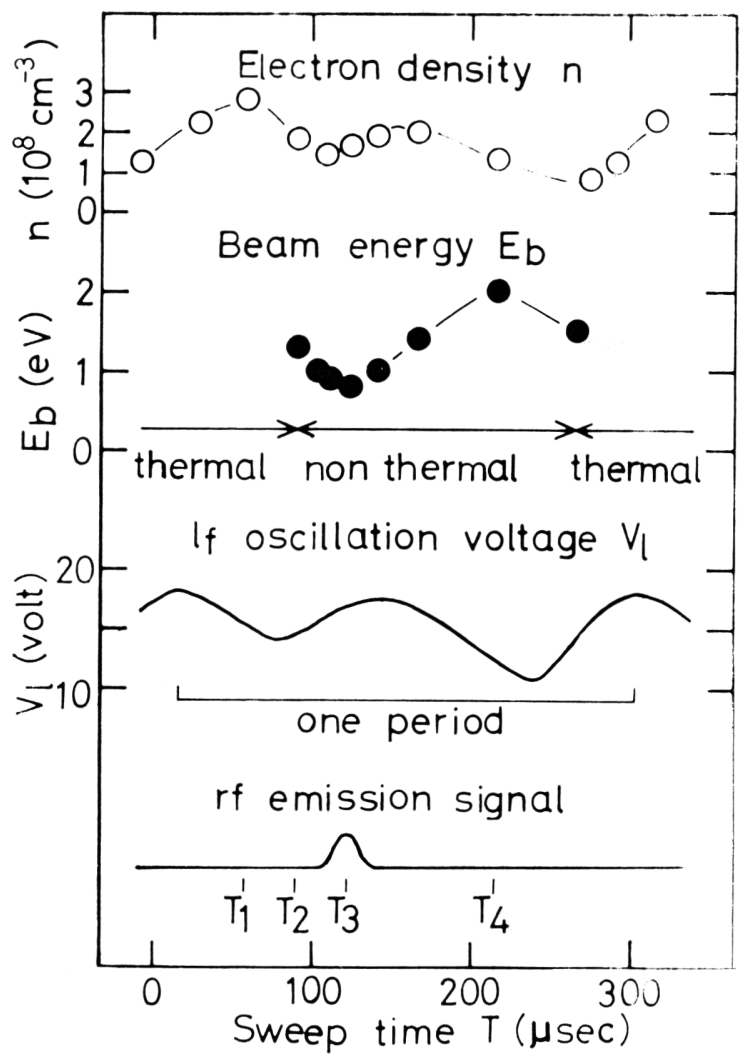

Fig. 5 The variation of beam energy and density of background electrons are shown together with an $\mathrm{rf}$ emission signal $(\mathrm{f}=371 \mathrm{MHz})$ and an lf oscillation voltage. The emission peak is about $2 \times 10^{-8}$ watt $\mathrm{f}_{c}=371 \mathrm{MHz} . \quad \mathrm{p}=1.83 \mathrm{mTorr} . \quad \mathrm{Id}=4.0 \mathrm{~mA}$.

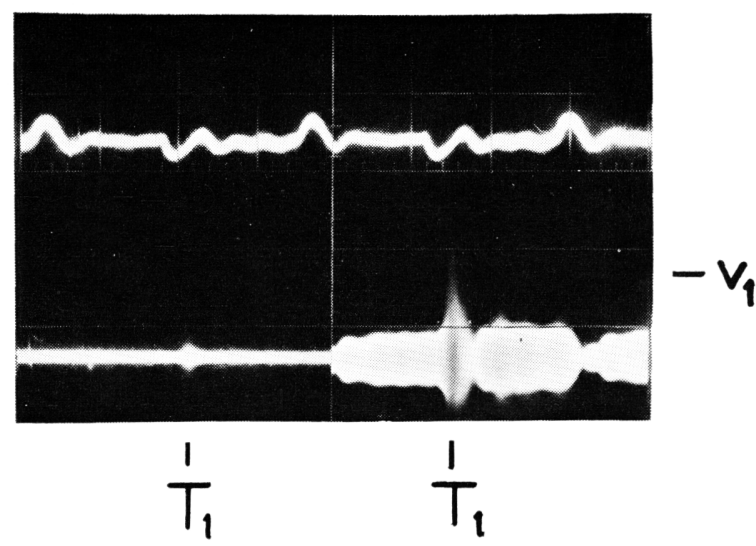

Fig. 6 Upper trace : If oscillation applied to an anode. 20 volt/div. Lowar trace: The part of left side shows the emission signal wthout incident signal, and one of the right shows tha output rf signal at an anode with incident signal. The signal level at the output of $\mathrm{rf}$ oscillator is denoted by $\mathrm{v}_{1}$. It is seen that $\mathrm{rf}$ signal is amplified at the phase $T_{1}$. Sweep time : $0.1 \mathrm{msec} /$ div. $P_{1}=-37 \mathrm{dBm}$ and $P_{2}=-33 \mathrm{dBm}$ at the phase $T_{1} \cdot \mathrm{p}=1.25 \mathrm{~m}$ Torr. $\mathrm{Id}=11.9 \mathrm{~mA}$ 


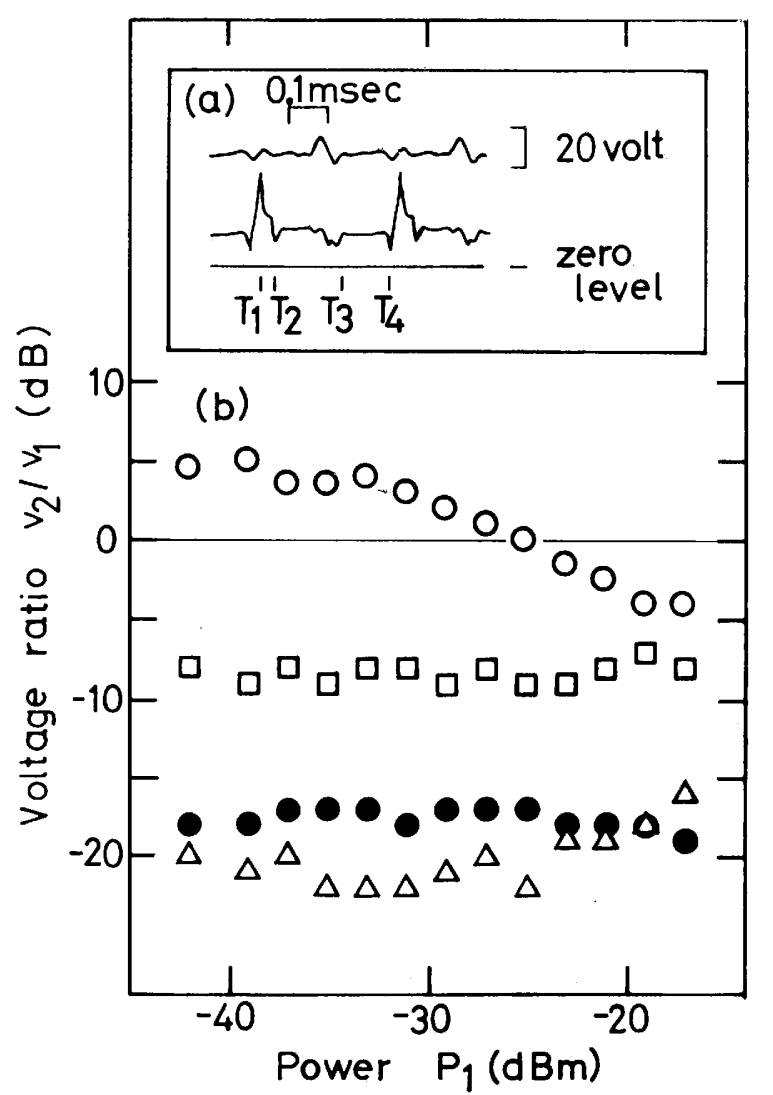

Fig. 7 (a) The phase where the transmission measurement was done are shown by $T_{1}, T_{2}, T_{3}$ and $T_{4}$ and related with an lf oscillation and an rf output signal at the anode

(b) Voltage ratio $\mathrm{v}_{2} / \mathrm{v}_{1}$ at each phase as a function of power level $P_{1} . f=230 \mathrm{MHz} . \mathrm{f}_{c}=236 \mathrm{MHz} . \mathrm{p}=1.25 \mathrm{~m}$ Torr. $\mathrm{Id}=11.9 \mathrm{~mA}$.

$O$, O"Uand $\Delta:$ at phase $T_{1}, T_{2}, T_{3}$ and $T_{4}$,

$\mathrm{dB}$, respectively. On a contrary, the ratio $\mathrm{v}_{2} / \mathrm{v}_{1}$ at the phase $\mathrm{T}_{1}$ had a nearly constant positive value of $5 \mathrm{~dB}$ at the weak incident power $\left(\mathrm{P}_{1}=-42 \sim-32 \mathrm{dBm}\right)$, decreased with $P_{1}$ when $P_{1}$ increased over the critical value $P_{c r i t}=-32 \mathrm{dBm}$ and became negative when $P_{1}$ exceeded $-25 \mathrm{dBm}$. This fact seems to imply that the negative absorption occurs at the phase $T_{1}$ only in the range of weak incident power and saturation phenomenon appears at strong incident power, as well as the experimental results verified for Xe plasma. ${ }^{7,8}$

We have shown the behaviours of negative absorption only at the electron cyclotron frequency $f_{c}$ in the previous paragraph. Next, its dependency on the frequency was investigated in the frequency region including $f_{c}$. The values of ratio $v_{2} / v_{1}$ as functions of frequency $f$ were measured at three phases denoted by $T_{1}, T_{2}$ and $T_{3}$, which are shown in Fig. 8 (a). The results are plotted in Fig. 8 (b). Three curves corresponding to the phases $T_{1}, T_{2}$ and $T_{3}$ show very different features each other near the electron cyclotron frequency. The curve corresponding to phase $T_{1}$ has the maximum of positive 


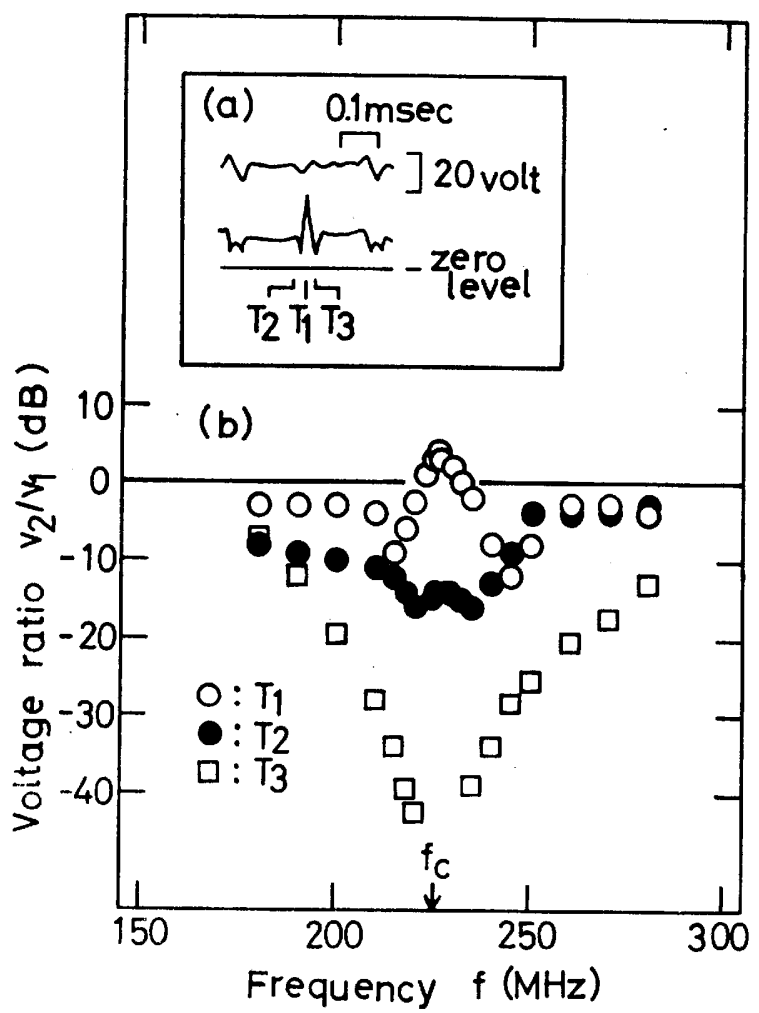

Fig. 8 The voltage ratios $v_{2} / v_{1}$ as functions of frequency $f$, at the phases $T_{1}, T_{2}$ and $T_{3}$, which are shown in (a) together with If oscillation voltage and $r f$ output signal in the case of $f=f_{c}$. $\mathrm{f}_{c}=226 \mathrm{MHz} . \mathrm{p}=1.25 \mathrm{mTorr}, \mathrm{Id}=11.9 \mathrm{~mA} . \mathrm{P}_{1}=-27 \mathrm{dBm}$

value at $f=f_{c}$, which gives the sufficient condition for negative absorption, while that corresponding to $T_{3}$ has the minimum of negative large value. However, in the region of frequency far from $f_{c}$, the three curves show the similar feature. Comparing with the theoretical consideration of the absorption coefficient in plasma, ${ }^{10,11}$ it is considered that the curve for $T_{3}$ shows the normal cyclotron absorption and the curves for $T_{1}$ and $T_{2}$ show the existence of the mechanism of negative absorption due to the stimulated cyclotron radiation. If the electrons are assumed to be monoenergetic, the effective collision frequency $\nu$ and the effective value of $h$ are estimated from the analysis of the feature of curve for $T_{1}$, which gives $\nu \approx 20 \mathrm{MHz}$ and $h \approx 3.7$. This value of $\mathrm{h}$ is considered to be much smaller than that obtained from the experimental data. ${ }^{12}$ This underestimation for $\mathrm{h}$ may come from the severe assumption that the electrons are monoenergetic.

It is verified from the experimental investigation described in this section that the negative absorption due to the stimulated cyclotron radiation does occur also in mercury plasma where the Ramsauer effect is very large. 


\section{Summary}

In the previous section has been described the investigation of the negative absorption phenomenon in weakly ionized mercury plasma, which is summarized as follows.

1) By applying the $\mathrm{rf}$ signal $\left(\mathrm{v}_{1}\right)$ to the grid, detecting at the anode the transmission signal $\left(v_{2}\right)$ and estimating the voltage ratio $v_{2} / v_{1}$, it is verified that negative absorption, i. e., the amplification of rf signal, occurs at the definite phase of if oscillation, when the frequency of the signal is nearly equal to the electron cyclotron frequency $f_{c}$.

2) The frequency region where this phenomenon is observed, is narrower than the elastic collision frequency $\nu$, i. e. $\left|f-f_{c}\right| \leqslant \nu$.

3) The ratio $v_{2} / v_{1}$ at the phase is nearly constant at the weak incident power $P_{1}$. However, it decreases with increasing $P_{1}$ over a certain value $P_{c r i t}$, so that the saturation effect is expected in this phenomenon.

4) If the parameters of plasma generation are adjusted to suitable values, the enormous emission pulse is observed synchronizing with an if oscillation at the electron cyclotron frequency, even when the rf signal is not applied from external source. Since the phase of If oscillation where this spontaneous emission pulse is observed does correspond to that where the negative absorption occurs at the slightly different condition of discharge, the emission pulse is considered to be self-oscillation which is excited by any mechanism of feedback through plasma.

5) The time-analyzed probe measurement at the phase of if oscillation where the emission pulse is observed shows that the beam component of electrons having the energy of about one electron volt exists in the nearly thermal background electrons. Since this value of electron beam energy situated near the value where the large Ramsauer effect is expected, the fact shows that the condition for the negative absorption may be satisfied at that phase.

It is concluded from the various experimental evidences, that the negative absorption due to the stimulated cyclotron radiation occurs in weakly ionized mercury plasma as well as $\mathrm{Xe}, \mathrm{Kr}$ and Ar plasmas.

\section{Acknowledgements}

The authors would like to express their sincere thanks to Professor S. Tanaka, Kyoto University for his interest and encouragement. They also thanks Mr. I. Kasuga, Kyoto University for his kindness to prepare the discharge tube used through the experiment. This work was partially supported by Grant-in-Aid from the Ministry of Education.

\section{References}

1) R. Q. Twiss, Australian J. Phys. 11, 564 (1956)

2) G. Bekefi, J. L. Hirshfield and S. C. Brown, Phys. Fluids 4, 173 (1961)

3) G. Bekefi, J. L. Hirshfield and S. C. Brown, Phy. Rev. 122, 1037 (1961)

4) S. Tanaka and K. Mitani, J. Phys. Soc. Japan 19, 1376 (1964) 
5) N. Shimomura, K. Mitani and S. Tanaka, J. Phys. Soc. Japan 21, 1372 (1966)

6) S. Tanaka, Y. Terumichi, K. Mitani and H. Kubo, J. Phys. Soc Japan 18, 1810 (1963)

7) Y. Terumichi, T. Idehara, I. Takahashi, H. Kubo and K. Mitani, J. Phys. Soc. Japan 20, 1705 (1965)

8) S. Tanaka and K. Takayama, J. Phys. Soc. Japan 21, 2372 (1966)

9) T. Idehara, J. Appl. Phys. 43, 64 (1972)

10) J. M. Wachtel and J. L. Hirshfield, Phys. Rev. Letters 19, 293 (1967)

11) G. Bekefi, Radiation Processes in Plasma (John Wiley and Sons, Inc., New York, 1966)

12) S. C. Brown, Basic Data of Plasma Physics (The M. I. T. Press, Massachusetts, 1966) 